\title{
Aplicabilidade da teoria de Orem na Assistência aos pacientes ostomizados
}

\author{
Applicability of Orem's theory in the care of ostomy patients \\ Aplicabilidad de la teoría de Orem en el cuidado de pacientes con ostomía
}

Cassiane da Silva Portela Pinto ORCID: https://orcid.org/0000-0002-5732-8555 Universidade do Estado do Pará, Brasil E-mail: cassiane_portela@yahoo.com Izadora Avelar Neto ORCID: https://orcid.org/0000-0002-3481-2088 Universidade do Estado do Pará, Brasil E-mail: izadoraavelar@gmail.com

Tatiana Menezes Noronha Panzetti ORCID: https://orcid.org/0000-0001-7560-4595 Universidade do Estado do Pará, Brasil E-mail: tnpanzetti@hotmail.com Alzinei Simor

ORCID: https://orcid.org/0000-0002-3848-5467 Universidade do Estado do Pará, Brasil E-mail: alzineisimor.enfo@gmail.com

Ingrid Magali de Souza Pimentel ORCID: https://orcid.org/0000-0003-1820-5496 Universidade do Estado do Pará, Brasil E-mail: imbarleta@yahoo.com

Carolyny Rosa Freire de Sá

ORCID: https://orcid.org/0000-0003-0705-5824 Universidade do Estado do Pará, Brasil

E-mail: carolynydrew@gmail.com

Lorena Vasconcelos Almeida Soares ORCID: https://orcid.org/0000-0001-7094-4021 Universidade do Estado do Pará, Brasil

E-mail: lorenavasconcelos13@gmail.com

Klebson Rodrigues da Silva

ORCID: https://orcid.org/0000-0003-3081-063X Universidade do Estado do Pará, Brasil E-mail: klebson.rdsilva@aluno.uepa.br

Caroline Martins Melo

ORCID: https://orcid.org/0000-0002-8280-6969 Universidade do Estado do Pará, Brasil E-mail: carolmartins0102@gmail.com Lais Gabriela da Silva Neves

ORCID: https://orcid.org/0000-0002-5200-5036 Universidade do Estado do Pará, Brasil E-mail: laisgneves18@gmail.com

Camila Andresa Monte Bezerra ORCID: https://orcid.org/0000-0002-4138-5299 Universidade do Estado do Pará, Brasil E-mail: montebezerra@hotmail.com

Catharina das Graças de Almeida Martins

ORCID: https://orcid.org/0000-0002-9353-1833 Universidade do Estado do Pará, Brasil

E-mail: catharina.amartins1@gmail.com

Hugo Vinicius Rodrigues da Silva ORCID: https://orcid.org/0000-0001-5332-7419 Universidade do Estado do Pará, Brasil E-mail: enf.hugo26@gmail.com

Marcos Antonio Ramos de Carvalho ORCID: https://orcid.org/0000-0001-9068-3017

Centro Universitário do Estado do Pará, Brasil E-mail: antoniio.ramoss10@gmail.com 


\section{Resumo}

Ostomia ou estoma representa a abertura feita em um procedimento cirúrgico para a exteriorização do intestino através da cavidade abdominal. O impacto das mudanças decorrentes dos estomas afeta diretamente a autoimagem, podendo ocasionar sentimento de tristeza, repulsa e insegurança diante da sensação de mutilação e incapacidade de ter uma vida normal. Nesse sentido, o presente artigo trata-se de uma revisão integrativa da literatura e tem como objetivo, verificar a aplicabilidade da Teoria de Orem aos pacientes ostomizados em estudos já realizados. Os pacientes ostomizados apresentam uma alteração na qualidade de vida, uma vez que o procedimento cirúrgico desencadeia mudanças na autoestima, na saúde mental e na rotina desses indivíduos. Nessa perspectiva, a Teoria de Orem se apresenta como uma ferramenta capaz de identificar os desvios de saúde do indivíduo, estabelecer intervenções baseadas nas Teorias dos Sistemas de Enfermagem e promover o autocuidado eficaz. Por fim, as ostomias comprometem não só as dimensões biológica, os padrões físiológicos e a autoimagem, mas também as relações psicossociais do indivíduo. Nesse contexto, a Teoria de Enfermagem de Orem é fundamental para os pacientes ostomizados, visto que engloba uma assistência holística ao sinalizar os desvios de saúde e classificar o sistema de enfermagem adotado em cada caso.
\end{abstract}

Palavras-chave: Estomia; Teoria de enfermagem; Autocuidado; Cuidados de enfermagem; Qualidade de vida; Autoimagem.

\begin{abstract}
Ostomy or stoma represents the opening made in a surgical procedure for the exteriorization of the intestine through the abdominal cavity. The impact of changes resulting from stomas directly affects self-image, which can cause feelings of sadness, repulsion and insecurity in face of the feeling of mutilation and inability to lead a normal life. In this sense, this article is an integrative literature review and aims to verify the applicability of Orem's Theory to ostomy patients in studies already carried out. Ostomized patients present a change in quality of life, since the surgical procedure triggers changes in self-esteem, mental health and routine of these individuals. From this perspective, Orem's Theory presents itself as a tool capable of identifying the individual's health deviations, establishing interventions based on the Theories of Nursing Systems and promoting effective self-care. Finally, ostomies compromise not only the biological dimensions, physiological patterns and self-image, but also the individual's psychosocial relationships. In this context, Orem's Nursing Theory is fundamental for ostomy patients, as it encompasses holistic care by signaling health deviations and classifying the nursing system adopted in each case.
\end{abstract}

Keywords: Ostomy; Nursing theory; Self-care; Nursing care; Quality of life; Self image.

\title{
Resumen
}

La ostomía o estoma representa la abertura que se realiza en un procedimiento quirúrgico para la exteriorización del intestino a través de la cavidad abdominal. El impacto de los cambios resultantes de los estomas incide directamente en la imagen de sí mismo, lo que puede provocar sentimientos de tristeza, repulsión e inseguridad ante el sentimiento de mutilación e incapacidad para llevar una vida normal. En este sentido, este artículo es una revisión integradora de la literatura y tiene como objetivo verificar la aplicabilidad de la Teoría de Orem a pacientes ostomizados en estudios ya realizados. Los pacientes ostomizados presentan un cambio en la calidad de vida, ya que el procedimiento quirúrgico desencadena cambios en la autoestima, la salud mental y la rutina de estos individuos. Desde esta perspectiva, la Teoría de Orem se presenta como una herramienta capaz de identificar las desviaciones de salud del individuo, estableciendo intervenciones basadas en las Teorías de los Sistemas de Enfermería y promoviendo un autocuidado efectivo. Finalmente, las ostomías comprometen no solo las dimensiones biológicas, los patrones fisiológicos y la autoimagen, sino también las relaciones psicosociales del individuo. En este contexto, la Teoría de Enfermería de Orem es fundamental para los pacientes ostomizados, ya que engloba el cuidado integral al señalar las desviaciones de salud y clasificar el sistema de enfermería adoptado en cada caso.

Palabras clave: Ostomía; Teoría de enfermería; Autocuidado; Cuidado de enfermera; Calidad de vida; Auto imagen.

\section{Introdução}

Ostomia ou estoma representa a abertura feita em um procedimento cirúrgico para a exteriorização do intestino através da cavidade abdominal (Rocha, 2011; Aguiar et al., 2019). Este processo cirúrgico objetiva o desvio temporário ou definitivo do conteúdo intestinal para uma bolsa coletora externa (Barbutti, 2008; Medeiros, 2017). Nesse sentido, os estomas realizados no intestino delgado (íleo) e intestino grosso (cólon), respectivamente, são as ileostomias que representam 12,6\% dos procedimentos realizados e as colostomias que constituem 82,7\% dos estomas (Rocha, 2011; Almeida, 2015).

As indicações dos estomas podem ocorrer por diversos motivos como obstruções intestinais, traumas, fístulas e a causa mais frequente a neoplasia colorretal (Rocha, 2011; Santos et al., 2007). Segundo o Instituto Nacional do Câncer 
(INCA), no ano de 2016 ocorreu um aumento na população de ostomizados de 34 mil novos casos (Medeiros, 2017). No que tange as mudanças sofridas pelos ostomizados, ocorrem tanto a nível fisiológico como também no nível psicossocial e emocional do paciente (Barbutti, 2008).

Nesse sentido, o impacto das mudanças decorrentes dos estomas afeta diretamente a autoimagem, provoca a perda da autoestima, o déficit no convívio social, devido a reclusão do paciente ostomizado e interferência no ciclo familiar podendo surgir o sentimento de incapacidade, inaceitação, repulsa e entre outros (Cascais, 2007; Coelho, Santos \& Pogetto, 2017). A recuperação dos fatores biopsicossocial e emocional é fornecida na atenção à saúde desses pacientes que deve abranger o estímulo ao autocuidado, a promoção da saúde e a prevenção de complicações (Oliveira, 2018).

$\mathrm{O}$ cuidado prestado aos pacientes ostomizados deve incluir o fornecimento de informações e a gestão do cuidado. Tais informações, referem-se aos cuidados da pele e da ostomia, a inclusão ou a exclusão de determinado alimento e o controle de gases e odores. Já a gestão do cuidado, deve avaliar as necessidades individuais e as atitudes do paciente em relação a ostomia. Além disso, a independência do indivíduo também deve ser estimulada de modo a permitir o autocuidado, bem como a identificação e a prevenção de possíveis complicações (Santos, Fava \& Dázio, 2019).

Nesse sentido, destaca-se a Teoria de Orem que é baseada em 3 pilares: o autocuidado, o déficit no autocuidado e os sistemas de Enfermagem. Enquanto a Teoria do Autocuidado versa sobre as práticas a serem realizadas tanto pelo indivíduo quanto pelos cuidadores, com o intuito de manter as condições vitais de saúde, a segunda aborda a atuação da enfermagem nas limitações ou incapacidades do autocuidado eficaz (Demoro et al., 2018). Já o ultimo pilar, estrutura e orienta as práticas profissionais através dos sistemas de enfermagens, sendo eles: o compensatório, parcialmente compensatório e o apoioeducação (Leone et al., 2021; Rodrigues, Bicalho \& Oliveira, 2019).

Diante disso, o objetivo desse trabalho é verificar a aplicabilidade da Teoria de Orem aos pacientes ostomizados.

\section{Metodologia}

Trata-se de uma revisão integrativa da literatura, cujo objetivo é verificar, expor e sintetizar os resultados de determinado tema a fim de discutir acerca das literaturas existentes e propor reflexões para a realização de novos trabalhos (Souza, Silva \& Carvalho, 2010; Bento, 2012). Essa metodologia é estruturada em 6 etapas: Na primeira etapa, é escolhida a pergunta norteadora: "Quais as evidências na literatura sobre a aplicabilidade da teoria de do autocuidado de Orem na Assistência de Enfermagem ao paciente com ostomia".

$\mathrm{Na}$ segunda etapa, foi realizado o levantamento de dados no período de abril a maio de 2021 nas seguintes plataformas: Literatura Latino Americana e do Caribe em Ciências da Saúde (LILACS), Scientific Electronic Library Online (SCIELO), National Library of medicine (PUBmed) e Medical Literature Analysis and Retrieval System Online (MEDLINE), foram utilizados os descritores "Teoria de enfermagem"; "Estomia"; "Autocuidado". Para a seleção dos artigos, foram aplicados os critérios de inclusão: literaturas em português, inglês e espanhol, no período de 2016 a 2021.

$\mathrm{Na}$ terceira etapa, foi realizada a catalogação dos artigos baseado na importância para o desenvolvimento do estudo, além de realizar confrontações entre as literaturas. Já na quarta e quintas etapas foram executadas com as interpretações e análises dos dados coletados. Por fim, na sexta etapa foi executada a condensação e a exposição de todos os estudos analisados.

Na primeira busca, foram encontrados 379 artigos na literatura, após a aplicação dos critérios de inclusão restaram 158 artigos. Desse total, foram feitas a leitura dos resumos, restando ao final 7 artigos. 
Figura 1. Fluxograma de seleção dos artigos sobre aplicabilidade da teoria de do autocuidado de Orem na Assistência de Enfermagem ao paciente com ostomia.

\section{Estudos encontrados nas bases de dados:}

LILACS ( $n=148)$ : Palavras-chave: Teoria de Enfermagem AND Estomia $(n=14)$; Teoria de Enfermagem AND Autocuidado ( $\mathrm{N}=288)$; Autocuidado AND Estomia $(\mathrm{n}=57)$.

PubMed: $(n=6)$ Palavras-chave: Teoria de Enfermagem AND Estomia $(n=6)$; Teoria de Enfermagem AND Autocuidado $(\mathrm{N}=0)$; Autocuidado AND Estomia $(\mathrm{n}=0)$.

Medline: $(n=0)$ Palavras-chave: Teoria de Enfermagem AND Estomia $(n=0)$; Teoria de Enfermagem AND Autocuidado $(\mathrm{N}=0)$; Autocuidado AND Estomia $(\mathrm{n}=0)$.

Scielo ( $n=14)$ Palavras-chave: Teoria de Enfermagem AND Estomia $(n=1)$; Teoria de Enfermagem AND Autocuidado $(\mathrm{N}=9)$; Autocuidado AND Estomia $(\mathrm{n}=4)$.

Total: $(n=379)$

Estudos rastreados: Trabalhos publicados entre 2016 a 2021, disponíveis nos idiomas português, inglês e espanhol

$$
\text { LILACS ( } \mathrm{n}=147)
$$

PubMed $(\mathrm{n}=1)$

Medline $(\mathrm{n}=0)$

Scielo $(\mathrm{n}=10)$

Total: (158)

Estudos escolhidos: aplicação dos critérios de inclusão

LILACS $(\mathrm{n}=6)$

PubMed $(\mathrm{n}=0)$

Medline $(n=0)$

Scielo $(\mathrm{n}=1)$

Total $=(n=7)$ $1^{\text {o }}$ exclusão: Trabalhos que não abordassem a temática

( $N^{o}$ de estudos excluídos:221) $2^{\circ}$ exclusão: Estudos excluídos a partir da leitura por não responder à pergunta norteadora do trabalho

( ${ }^{\circ}$ de estudos excluídos: 151)

Fonte: Autores. 


\section{Resultados e Discussão}

No que diz respeito ao ano de publicação, foram encontrados um artigo em 2021 e dois nos anos de 2019,2017 e 2016 todos de Língua Portuguesa. Quanto ao delineamento dos estudos, foram identificados três estudos descritivos e exploratórios, duas revisões integrativas da literatura, um estudo transversal, além de um estudo de método misto. Esses trabalhos foram sintetizados no quadro 1, segundo título, ano, país, delineamento e demandas assistenciais.

Quadro 1. Síntese dos artigos incluídos na revisão por título, ano de publicação, delineamento e demandas assistenciais, Belém (PA), Brasil, 2019

\begin{tabular}{|c|c|c|c|}
\hline Título & $\begin{array}{l}\text { Ano } \\
\text { País }\end{array}$ & Delineamento & Demandas assistenciais \\
\hline $\begin{array}{c}\text { Assistência de enfermagem em } \\
\text { diálise peritoneal: aplicabilidade da } \\
\text { teoria de orem - estudo de método } \\
\text { misto }\end{array}$ & $\begin{array}{c}2021 \\
\text { Brasil }\end{array}$ & $\begin{array}{l}\text { Estudo de método } \\
\text { misto }\end{array}$ & $\begin{array}{l}\text { - Identificação dos desvios de autocuidado em } \\
\text { pacientes em diálise peritoneal, as necessidades } \\
\text { de cuidado e a intervenção para cada caso } \\
\text {-Redução de risco e de sofrimento } \\
\text {-Elevação da qualidade do cuidado e a } \\
\text { visibilização do enfermeiro }\end{array}$ \\
\hline $\begin{array}{l}\text { Self-care of elderly people with } \\
\text { ostomy by colorectal cancer }\end{array}$ & $\begin{array}{c}2019 \\
\text { Brasil }\end{array}$ & $\begin{array}{l}\text { Revisão } \\
\text { integrativa de } \\
\text { literatura }\end{array}$ & $\begin{array}{l}\text {-Ajudar na adaptação da nova realidade, na } \\
\text { melhoria do autocuidado com a ostomia e no } \\
\text { suporte informacional e emocional } \\
\text { - Ajudar a enfrentar as mudanças na autoimagem } \\
\text { e na perda do controle do esfincter }\end{array}$ \\
\hline $\begin{array}{c}\text { Estratégias de Atendimento } \\
\text { Psicológico a Pacientes Estomizados } \\
\text { e seus Familiares }\end{array}$ & $\begin{array}{c}2019 \\
\text { Brasil }\end{array}$ & $\begin{array}{l}\text { Estudo descritivo } \\
\text { e exploratório }\end{array}$ & $\begin{array}{l}\text {-Necessidade de acolhimento psicológico para } \\
\text { minimizar sofrimento } \\
\text { - Ajudar a desmitificar o processo de estomização } \\
\text { - Fornecer assistência focada no } \\
\text { desenvolvimento de competências de } \\
\text { autocuidado. }\end{array}$ \\
\hline $\begin{array}{l}\text { Oncology ostomized patients' } \\
\text { perception regarding sexual } \\
\text { relationship as an important } \\
\text { dimension in quality of life }\end{array}$ & 2017 Brasil & $\begin{array}{l}\text { Estudo transversal } \\
\text { e descritivo }\end{array}$ & $\begin{array}{l}\text {-Ajudar na percepção da autoimagem, na baixa } \\
\text { autoestima, na realização de atividades diárias e } \\
\text { na função sexual prejudicada }\end{array}$ \\
\hline $\begin{array}{l}\text { As práticas educativas e o } \\
\text { autocuidado: evidências na produção } \\
\text { científica da enfermagem }\end{array}$ & $\begin{array}{c}2016 \\
\text { Brasil }\end{array}$ & $\begin{array}{l}\text { Revisão } \\
\text { integrativa da } \\
\text { literatura }\end{array}$ & $\begin{array}{l}\text { - Ações de autocuidado inovadoras } \\
\text {-Promoção do autocuidado }\end{array}$ \\
\hline $\begin{array}{c}\text { Autocuidado: uma estratégia para a } \\
\text { qualidade de vida da pessoa com } \\
\text { estomia }\end{array}$ & $\begin{array}{c}2016 \\
\text { Brasil }\end{array}$ & $\begin{array}{l}\text { Pesquisa } \\
\text { descritiva }\end{array}$ & $\begin{array}{l}\text { - O autocuidado garantindo uma qualidade de } \\
\text { vida } \\
\text {-Enfermagem promotora do autocuidado }\end{array}$ \\
\hline $\begin{array}{c}\text { Autoimagem e autocuidado na } \\
\text { vivência de pacientes estomizados: o } \\
\text { olhar da enfermagem }\end{array}$ & $\begin{array}{c}2017 \\
\text { Brasil }\end{array}$ & $\begin{array}{l}\text { Estudo } \\
\text { exploratório } \\
\text { descritivo e } \\
\text { qualitativo }\end{array}$ & $\begin{array}{l}\text {-relação ao autocuidado e aceitação } \\
\text { do paciente com um olhar diferenciado } \\
\text { - Auxiliar as pessoas estomizadas a encarar essa } \\
\text { experiência } \\
\text {-melhorar a adaptação e melhor qualidade de } \\
\text { vida dos estomizados. }\end{array}$ \\
\hline
\end{tabular}

Fonte: Autores.

Identifica-se na literatura que os pacientes ostomizados apresentam uma alteração na qualidade de vida, uma vez que o procedimento cirúrgico desencadeia mudanças na autoestima, na saúde mental e na rotina desses indivíduos. Nessa perspectiva, a Teoria de Orem se apresenta como uma ferramenta capaz de identificar os desvios de saúde do indivíduo, estabelecer intervenções baseadas nas Teorias dos Sistemas de Enfermagem e promover o autocuidado eficaz.

Em relação a autoestima, esse desvio de saúde ocorre pela mudança corporal, pela diminuição da percepção de atração sexual e pelo isolamento social - explicado pelos julgamentos da sociedade e pelo medo de vazar efluentes da bolsa - (Santos, Fava \& Dázio, 2019; Kimura et al., 2017). 
Além disso, o uso das ostomias afeta os "padrões de beleza" social, pois esses pacientes são desvinculados do corpo bonito, saudável e higiênico provocando alterações significativas na autoestima e autoimagem do paciente e em uma cascata de eventos afeta seu estado nutricional, físico, social, sexual e principalmente psicológico, levando o indivíduo a se isolar ou em alguns casos entrar em quadro depressivos. (Freire et al., 2017; Mota et al, 2016)

Dessa forma, a Teoria de Orem nos requisitos para o autocuidado por desvio de saúde tem grande influência nesse contexto, visto que esta busca a adaptação do indivíduo a uma determinada realidade, a exemplo, a modificação do autoconceito e da autoimagem decorrente da patologia. Faz-se necessário que a enfermagem desempenhe e promova estratégias de autoaceitação o que se configura como fator essencial no enfrentamento de sua nova condição de vida.

Partindo dessa premissa, as intervenções devem envolver o sistema de Enfermagem parcialmente compensatório, entendido pelo cuidado proveniente da equipe de enfermagem e do paciente (Leone et al., 2021), visto que o profissional deve fornecer informações de forma clara e objetiva para que o indivíduo entenda e desenvolva habilidades para lidar com as suas mudanças excretoras (Santos, Fava \& Dázio, 2019; Kimura et al 2017). Além disso, esse esclarecimento deve envolver as questões referentes a sexualidade, como a disfunção sexual e a mudança corporal para criar estratégias de enfrentamento e promover uma vida sexual ativa e agradável (Kimura et al., 2017).

Os pacientes ostomizados sofrem mudanças significativas no seu estilo de vida, como mudanças físicas, emocionais, social, econômica, familiar, ocorrem também mudanças em seus hábitos e costumes, o que na maioria das vezes afeta sua qualidade de vida e autoestima (Mota et al., 2016). Essas modificações no estilo de vida do paciente afetam também os requisitos da Teoria do Autocuidado, pois interferem na manutenção e funcionamento do bem-estar do indivíduo, por vezes não tendo a prevenção de risco à vida.

No que diz respeito à saúde mental, é comum que a ostomia desencadeie tristeza, repulsa, sensação de mutilação e de incapacidade de ter uma vida normal. E como forma de solucionar essa problemática, os artigos sugerem o acolhimento emocional, o fortalecimento de estratégias de enfretamento e o desenvolvimento do protagonismo do cuidar (Silva et al., 2019).

Esses mecanismos são alcançados a partir do entendimento dos seus sentimentos e do desenvolvimento de estratégias para lidar com esse cenário. Tal argumento está em consonância com o Sistema de Apoio-educação que ocorre quando o indivíduo é capaz de realizar o autocuidado, porém com limitações e precisa de orientações da equipe (Leone et al., 2021).

$\mathrm{Na}$ análise das literaturas, identificou-se que a participação do paciente ostomizado em grupos terapêuticos são estratégias para a promoção do autocuidado, uma vez que os encontros em grupos trabalham a autonomia e independência do paciente por meio de um processo continuo. Ademais, os estudos mostram que a realização de práticas educativas e de educação em saúde desenvolvidas pelo enfermeiro, envolvendo a comunidade e os familiares se tornam essenciais no processo do autocuidado (Tossin et al., 2016).

Nesse sentido, na Teoria de Sistemas de Enfermagem, especificamente no princípio da enfermagem em seu trabalho, atribui ao enfermeiro a função de realizar a assistência com abordagens familiares, estimulando e levando o paciente a participação de grupos como pratica de autocuidado e como maneira do paciente atuar na melhoria da sua qualidade de vida.

\section{Considerações Finais}

Com o presente trabalho, foi possível observar que as ostomias comprometem não só as dimensões biológica, os padrões fisiológicos e a autoimagem, mas também as relações psicossociais do indivíduo. Além disso, destaca-se o importante papel da enfermagem na orientação aos pacientes ostomizados sobre o enfrentamento da sua nova realidade, oferecendo apoio psicológico, ajudando no processo de autoaceitação e assumindo um papel de facilitador durante todo o processo de cuidado com esses pacientes. 
Nesse contexto, a Teoria de Enfermagem de Orem é fundamental para os pacientes ostomizados, visto que engloba uma assistência holística ao sinalizar os desvios de saúde e classificar o sistema de enfermagem adotado em cada caso. Tais praticas, garantem uma redução de riscos, a diminuição do sofrimento e uma melhora tanto na qualidade de vida do paciente quanto no cuidado ofertado. Por fim, faz-se necessário incentivar a produção cientifica sobre a temática abordada, visto as poucas evidencias na literatura.

No mais, destaca-se a importância de novos estudos sobre o tema, dada as restritas produções literárias e a relevância da teoria para elaborar planos assistenciais adequados, holísticos e humanizado.

\section{Referências}

Aguiar, F. A. S., Jesus, B. P., Rocha, F. C., Cruz, I. B., Neto, G. R. A., Rios, B. R. M., Piris, A. P., \& Andrade, D. L. B. (2019). Colostomia e autocuidado: significados por pacientes estomizados. Rev. enferm. UFPE on line, 105-110.

Almeida, E. J., \& Silva, A. L. (2015). Caracterização do Perfil Epidemiológico dos Estomizados em Hospitais da Secretaria de Estado de Saúde do Distrito Federal. Estima-Brazilian Journal of Enterostomal Therapy, 13.

Barbutti, R. C. S., Silva, M. C. P. \& Abreu, M. A. L. (2008). Ostomia, uma difícil adaptação. Revista da SBPH, 11(2), 27-39.

Bento, A. V. (2012). Como fazer uma revisão da literatura: Considerações teóricas e práticas. Revista JA (Associação Académica da Universidade da Madeira), 7(65), 42-44.

Cascais, A. F. M. V; Martini, J. G., \& Almeida, P. J. S. (2007). Ostomy impact in the process of human living. Texto \& Contexto-Enfermagem, 16(1), 163167.

Coelho, A. R., Santos, F. S., \& Poggetto, M. T. S. (2013). A estomia mudando a vida: enfrentar para viver. Revista Mineira de Enfermagem, $17(2), 258-277$.

Demoro, C. C. S., Fontes, C. M. B., Trette, A. S., Cianciarullo, T.I., \& Lazarini, I. M. (2018). Applicability of Orem: training of caregiver of infant with Robin Sequence. Rev. Bras. Enferm., 71(3), 1469-1473 <http://www.scielo.br/scielo.php?script=sci_arttext\&pid=S0034-71672018000901469\&lng=en\&nrm=iso>.

Freire, D. A., Angelim, R. C. M., Souza, N. R., Brandão, B. M. G. M., Torres, K. M. S., \& Serrano, S. Q. (2017). Autoimagem e autocuidado na vivência de pacientes estomizados: o olhar da enfermagem. Revista Mineira de Enfermagem, 21.

Kimura, C. A., Guilhem, A. B., Kamada, I., Abreu, B. S., \& Fortes, R. C. (2017) Oncology ostomized patients' perception regarding sexual relationship as an important dimension in quality of life. J. Coloproctol, 37(3), 199-204.

Leone, D. R. R., Neves, A. C. O. J., Prado, R. T., \& Castro, E. A. B. (2021). Assistência de enfermagem em diálise peritoneal: aplicabilidade da teoria de orem - estudo de método misto. Esc. Anna Nery, Rio de Janeiro, v. 25, n. 3, p.1-9.

Medeiros, L. P., Silva, I. P., Lucena, S. K. P., Sena, J. F., Mesquita, E. K. S., Oliveira, D. M. S., \& Cota, I. K. F. (2017). Atividades da intervenção de enfermagem cuidados com a ostomia. Rev. enferm. UFPE on line, 5417-5426.

Mota, M.S., Gomes, C. G; Silva, C.D., Gomes, V. L. O., Pelzer, M. T., \& Barros, E. J. L. (2016). Autocuidado: uma estratégia para a qualidade de vida da pessoa com estomia. Investigación en Enfermería: Imagen y Desarrollo, 18, 63-78.

Oliveira, I. V., Silva, M. C; Silva, E.L; Freitas, V. F; Rodrigues, F. R; \& Caldeira, L. M. (2018) Cuidado e saúde em pacientes estomizados. Revista Brasileira em Promoção da Saúde, 31(2).

ROCHA, J. J. R. (2011) Estomas intestinais (ileostomias e colostomias) e anastomoses intestinais. Medicina (Ribeirão Preto), $44,51-56$.

Rodrigues, H. A., Bicalho, E. A. G., \& Oliveira, R. F. (2019) Cuidados de enfermagem em pacientes ostomizados: uma revisão integrativa de literatura. Psicologia e Saúde em debate, 5, 110-120.

Santos, C. H. M., Bezerra, M. M., Bezerra, F. M. M., \& Paraguassú, B. R. (2007). Perfil do paciente ostomizado e complicações relacionadas ao estoma. Revista Brasileira de Coloproctologia, 27, 16-19.

Santos, R.P., Fava, S. M. C. L., \& Dazio, E. M. R. (2019). Self-care of elderly people with ostomy by colorectal cancer. J. Coloproctol. 39(3), 265-273

Silva, N. M., Santos, M. A., Barroso, B. C. T., Rosado, S. R., Teles, A. A. S., \& Sonobe, H. M. (2019). Estratégias de Atendimento Psicológico a Pacientes Estomizados e seus Familiares. Psicol. cienc. prof., 39, 1-16.

Souza, M. T., Silva, M. D. S., \& Carvalho, R. (2010) Revisão integrativa: o que é e como fazer. Revista Einstein., 8, $102-106$.

Tossim, B. R., Souto, V. T., Terra, M. G., Siqueira, D. F., Mello, A. L., \& Silva, A. A. (2016) As práticas educativas e o autocuidado: evidências na produção científica da enfermagem. Revista Mineira de Enfermagem 20. 